\title{
The Effects of Bitter Placebos on Cognitive Tests
}

\author{
Scott L. Kemsley, Chi Man Leung, Suet Mui Ma, Samantha C. Lam, Boyd H. Timothy, \\ Ronald M. Miller
}

Department of Psychology, Brigham Young University, Hawaii, USA

\section{Email address:}

bellechiman@gmail.com (C. M. Leung), evelyndot106@gmail.com (S. M. Ma), celestinalam@gmail.com (S. C. Lam), boyd.timothy@gmail.com (B. H. Timothy),dr.ronald.miller@gmail.com (R. M. Miller)

\section{To cite this article:}

Scott L. Kemsley, Chi Man Leung, Suet Mui Ma, Samantha C. Lam, Boyd H. Timothy, Ronald M. Miller. The Effects of Bitter Placebos on Cognitive Tests. Psychology and Behavioral Sciences. Vol. 5, No. 4, 2016, pp. 98-103. doi: 10.11648/j.pbs.20160504.14

Received: June 11, 2016; Accepted: July 5, 2016; Published: July 7, 2016

\begin{abstract}
Placebo effect has been applied in multiple aspects and the different components of placebo effect have already been studied in many different researches. Taste, which is one of the physiological effects, is a vital component of the placebo effect, and according to a Chinese idiom, "bitter taste medicines are more effective", bitter tastes comes into play. However, bitter placebo does not work for everyone because of cultural difference and taste preferences. Results from factorial ANOVA showed there was a significant interaction effect of the taste of the drink and the type of personality on cognitive test performance when it came to speakers of different languages: native English and non-native English speaking participants. Also, bitter placebo only works for supertasters who have experience of the aversion of bitterness and agree that bitter drinks can keep themselves healthy. Hence, supertaster participants who feel gross after tasting the bitter drink will persuade themselves the benefit of the placebo. For future studies, it should modify the methods with a self-rated scale of the placebo taste and a different placebo substance with a universal flavor for improvement.
\end{abstract}

Keywords: Placebo Effect, Supertaster, Non-supertaster, Bitterness, Taste Preferences, Cognitive Test

\section{Introduction}

Effects of any treatment can be divided into two categories: characteristic effect and incidental effect, also known as the placebo effect [1]. According to Levine, Gordon, and Fields (1978), characteristic factors are defined as "therapeutic actions or strategies that are theoretically derived, unique to a specific treatment, and believed to be causally responsible for the outcome", and placebo factors are defined as "the many other factors that have also been shown to affect outcomes, such as the credibility of the intervention, patient expectations, the manner and consultation style of the practitioner, and the therapeutic setting".

Placebo has been existed as long as medicine itself, as ancient medication had more of a psychological effect than a physical effect [2]. The placebo effect has been studied much more rigorously recently in modern medicine, psychology, and related fields. According to Beecher (1995), the placebo effect can actually help aid, and even cure multiple conditions, including anxiety and sleeping disorder [3].
However, the full term "placebo effect" was not used until 1906 in Cabot's "The Physician's responsibility for the Nostrum Evil" [4]. Cabot suggested that the placebo effect was deceptive. Before the mid-1950s, most researchers' views followed Cabot's ideas and only referred to the placebo effect in terms of error variance, as it tended to complicate and skew the data. Yet more modern researchers have noted that the placebo effects could actually contribute to other causes [5]; [6], and Beecher's ground-breaking study (1955) allowed for an outbreak of additional studies on the placebo effect, weaning away from the previous error variance view [3].

The effect of placebo can be applied in multiple aspects, and different components of placebo effect have already been studied in different researches. For example, the cost of the placebo has a significant consequence on its effectiveness [7]. In the study, all participants received a placebo pill that was given to prevent pain. Half of the participants were told that their pills were purchased at a discount $(\$ 0.10$ per pill 
instead of the normal price of $\$ 2.50$ ). They were then administered a series of electric shocks, once before and once after taking the placebo. Participants who were administered the full-price placebo expressed that the placebo was more significantly effective in reducing pain than the participants who were given the placebo bought at a discount price expressed.

Other than the cost of placebo, color and odor are other examples of different components of the placebo effect. A few studies have been conducted on the color effect of placebos. In a study, 34 participants were given a yellow placebo pill [8]. The participants indicated that the placebo triggered them to be more energetic with an increase in appetite. Another study, conducted by Schindel (1978), participants in the study reported better effectiveness of the placebo of their preferred color's placebo pills [9]. In a study conducted by Jacobs and Nordan (1979), participants handled, but did not take, red, yellow, green, blue, black, and white capsules and were asked if they appeared to be depressants, stimulants, or hallucinogenic drugs [10]. The results indicated that the participants viewed the blue pills as depressants, and red and yellow pills as stimulants, complementing with Lasagna, Laties and Dohan's study (1958) on yellow placebos [8]. Odor can also have a positive or adverse effect on mood, which can be a major variable in the placebo effect. In a study conducted by Marchand and Arsenault (2002), it revealed that pleasant odors can alleviate pain, and unpleasant odors can increase pain in women [11]. Both color and odor are under the field of physiological effect, which is one of the vital components of the placebo effect that was defined by [12]. In essence, physiological effect is treatment effect that caused by the physical, often sensual, properties of the placebo. Smell, viscosity, acidity, temperature, texture, and taste are some of the examples of the physiological effect.

With the idea of the physiological effect and the multiple factors that affect the effectiveness of a placebo, the Chinese idiom “苦口良藥”, which translates to “bitter taste medicines are better/ more effective", comes into play. A research suggests that one of the two major ways that nonhuman primates self-medicate is by partaking bitter pith and leaves, which leaves some to question whether this self-medication is physical or psychological [13]. Mennella, Spector, Reed, and Coldwell (2013) also suggested that "the more potent the drug, the more bitter it will be" [14]. However, no peer-reviewed research has looked at the effect of bitterness, specifically, on the strength of the placebo effect.

Although taste has its placebo effect on human, different ethnicities have their own taste preferences, and may react differently even using the same taste of the placebo. According to Mennella, Pepino, and Reed (2005), genetic variations influence human's taste genes, and cultural differences have resulted in different sensitivity in bitter taste and preferences of sweet taste [15]. In the study, participants of African ancestry have a sweeter taste preference than Caucasian participants. The researchers also suggested that cultural forces and experiences may determine the result of overriding the genotype effect on individuals' sweet preference.

Apart from the effect of ethnicity on individuals' taste preferences, genetic factor also impacts on taste preferences by developing different amounts of fungiform papillae on tongue. Those who have a denser covering of fungiform papillae were called as the "supertasters" [39], which means they are able to distinguish smaller changes in ingredient levels [16] and perceive more nuanced flavors [39]. Several studies have shown that supertasters often perceive common food as too sweet, spicy, or bitter. For example, they found low concentration of caffeine is too bitter, and ice-cream is too sweet for them than for non-supertasters [17], [39].

Multiple variables of the placebo have been discussed, however, the variables of the participants have yet to be considered. According to Duncan and Laird (1980), individuals respond differently even to the same condition by using different types of cues to make attribution of themselves, and the two types of cues are self-produced cue, and situational cue [18]. Self-produced cues are defined as cues created from an individual's activities. These include overt actions, statements of belief, expressive behavior, and arousal. Contrarily, situational cues were defined as cues taken from the situation that influences the individual's feelings.

A study of Stevens (1996), "Individual differences in taste perception", supported the idea of different self-perceptive cues [19]. In the study, a placebo was used, and its tastes were the variables. Participants in the study were given chicken soup with different levels of sodium concentration: $0.276 \%$ (normal), $0.420 \%$ (high), or $0.564 \%$ (very high), and each of them was attached with labels "less than normal flavoring", "more than normal flavoring", or nothing. The result of the study showed that self-produced cue users were more sensitive to the changes in sodium concentration while situational cue users were more focus on the labeling.

By linking all the information from the researches on selfperceptive cues and "supertaster", self-produced cue users fit into the characteristic as supertasters, not because selfproduced cue users have a denser covering of fungiform papillae on their tongues, but as self-produced cue users are more aware of their bodies' cues. They are more sensitive in the sense of taste. In contrast, situational cue users are less sensitive to their bodies' cues. They fit into the "nonsupertaster" category.

In looking at the studies related to the placebo effect, bitter medicine, supertaster, and self-perceptive cues, it is hypothesized that a) self-produced cue dominant individuals (supertasters) will perform better on cognitive tests after drinking a bitter placebo, and b) situational cue dominant individuals (non-supertasters) will perform better on cognitive tests after drinking sweet placebos.

\section{Methods}




\subsection{Participants}

The participants consisted mostly young adults $\left(\mathrm{M}_{\text {age }}=\right.$ 21.48 years, age range: 18-35 years) who were recruited through flyers and face-to-face recruitment at a multi-cultural Western university. Many participants were compensated for their participation with extra-credit for their psychology courses. There were 65 male participants and 87 female participants, for a total of 152 participants. Of these 152 participants, 91 reported the United States as their home region, 32 reported Asia and 13 reported the Pacific Islands, leaving 16 participants claiming other regions. However, only 151 out of 152 participants reported their native languages. 103 participants selected English as their first language while 48 participants indicated that they were not native English speakers.

\subsection{Materials and Procedure}

First, an experiment of the tastes of wild chamomile tea was conducted in order to test individual's taste sensitivity to bitterness and sweetness. As a result, Caucasians had observable differences when they were responding to sweet and unsweet wild chamomile tea. Therefore, wild chamomile tea was adopted as a practical material to test individual's taste preference on the cognitive tests.

In the actual experiment, participants were first given an Expression-Manipulation Procedure (EMP) [18], [19]. in order to determine whether they were highly sensitive to taste. EMP introduces eight different emotions including anxious, interested, angry, sad, happy, disgusted, afraid, and surprised. The adaptation of EMP was based on the theory of emotion of James (1884) [20]. Theorists addressed that the way people decided their own moods and feelings was the same as the way they decided them in others [19], [21]. Stevens (1996) further emphasized that some people had strong emotional fluctuations along their regulation of facial expression while some did not [19]. It was so because of the differences of dominant personalities. Self-produced cue users were sensitive or aware of inner personal feelings or emotional expressions, whereas situational cue users were likely to be affected by outward components such as environments or people. At the same time, Stevens also described supertasters as self-produced cue users and nonsupertasters as situational cue users because supertasters/ self-produced cue users were more sensitive to inner bodily feelings such as the mouth movements (e.g., chewing, salivation, swallowing and sniffing) while non-supertasters/ situational cue users focused more on outer stimuli such as information from previous personal or cultural experience [19].

In the EMP test, participants were asked to adopt certain facial features and express how each facial feature affected different elements of their mood. Participants were specifically asked to adopt a smile and frown twice, interchangeably. Then, they were asked to rate how they felt based on the eight given emotions. Supertaster personality were significantly more affected by applying the given facial features than non-supertaster personality.

Next, participants were randomly assigned to a sweetened (condition) or unsweetened wild chamomile tea (condition). Four grams of chamomile herbs were boiled in a pot of two gallons of water and then poured into two one-gallon pitchers. The first pitcher was left unchanged and marked as unsweetened, while $1 \frac{1}{2}$ cups of sugar was placed into the second. Each participant receiving either form of the tea was told that the tea would increase blood flow to the parts of the brain corresponding with oration, which should help them with cognitive test performance. This declaration was stated as fact for purposes of this study, but there is no empirical evidence suggesting that chamomile, sweetened or otherwise, has any effect on verbal acuity.

All participants were asked to complete a verbal GRE test in order to assess their cognitive performance. The higher GRE score they earned, they higher ability of analysis and evaluation of written material indicated. The verbal GRE test was designed to assess participants' integration of information obtained from it, analysis of relationships among component parts of sentences, and recognition of relationships among words and concepts. It was also designed to be taken by individuals who have completed an undergraduate degree and are applying for graduate programs. The verbal GRE section was then administered to all participants. Sixteen questions were posed to the participants. All questions were obtained from previous GRE exams. The first five questions were vocabulary-based in which the participants were asked to use context clues to fill in blanks within a sentence with the appropriate vocabulary term. The second section required participants to read a short article and then answer multiple choice questions that assessed their comprehension of the article. The next section consisted of three analogies that tested vocabulary along with association skills. Finally, the last four questions required reading an additional article and, again, answering comprehension questions about that article.

\section{Results}

In order to examine whether bitter placebo has an effect on supertasters and non-supertasters' cognitive test performance, data of 151 participants were collected. The result showed that supertasters $(M=8.03)$ scored higher than non-supertasters $(M=7.84)$ when given bitter drink. Supertasters also $(M=7.87)$ scored higher than nonsupertasters $(M=7.51)$ when given sweeten drink. However, there was no significant effect of the taste of drink, $F(1,151)$ $=.26, p=.61, \eta_{p}{ }^{2}=.00$. There was no statistically significance of the effect of the type of personality, $F(1,151)$ $=.03, p=.87, \eta_{p}{ }^{2}=.00$. There was no significant interaction of the tastes of drink and the type of personality on test score, $F(1,151)=.17, p=.68, \eta_{p}{ }^{2}=.00$. 


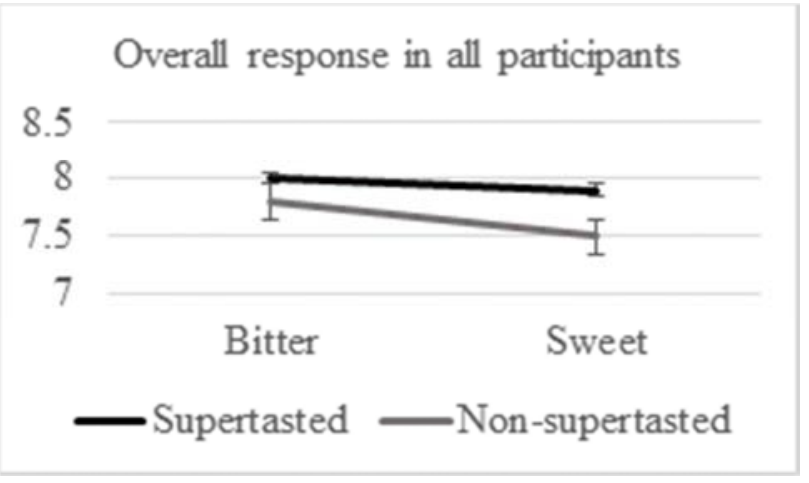

Figure 1. Overall effect of bitter and sweet drink and cue responses on all participants' GRE score performance.

To further test the effect of language differences on test performance, data was split into English speaking and nonEnglish speaking groups. Results showed that English speaking supertasters $(M=9.80)$ scored higher than English speaking non-supertasters $(M=8.48)$ when they were given bitter drink. English speaking non-supertasters scored higher $(M=9.57)$ than English speaking supertasters $(M=8.33)$ when they were given sweetened drink. For English speaking participants, there was no significant difference of the effect of the taste of the drink, $F(1,103)=.17, p=.68, \eta_{p}{ }^{2}=.00$. It was not significant for the effect of the types of personality on cognitive test performance as well, $F(1,103)=.007, p$ $=.93, \eta_{p}{ }^{2}=.00$. Yet, there was significant for the interaction between that taste of the drink and the type of personality, $F(1,103)=7.85, p=.01, \eta_{p}{ }^{2}=.07$.

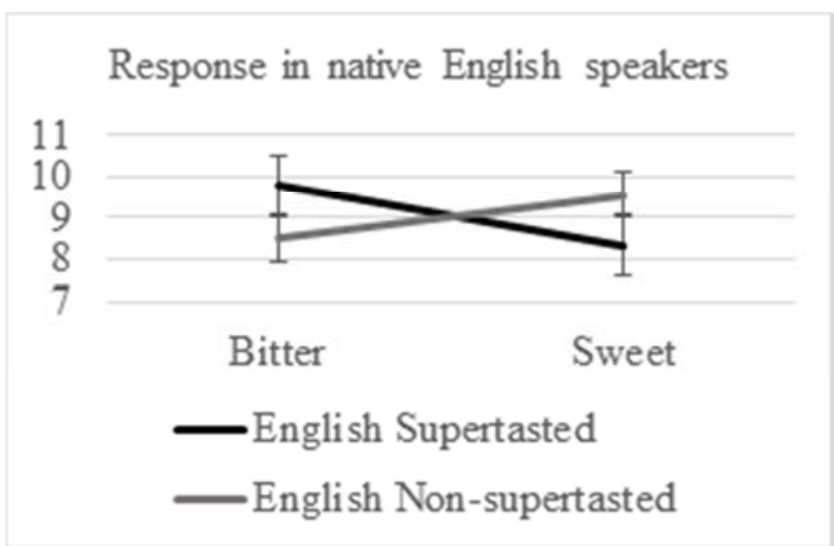

Figure 2. The effect of bitter and sweet drink and cue responses on native English speakers' GRE score performance.

For non-English speakers, non-supertasters $(M=6.31)$ scored higher than supertasters $(M=4.50)$ when given bitter drink; supertasters $(M=6.00)$ scored higher than nonsupertasters $(M=4.47)$ when they were given sweet drink. The weighted result showed that there is significance of the interaction between the taste of the drink and the type of personality to both English speaking participants and nonEnglish speaking participants. The result shows that nonEnglish speakers, there was no statistical significance of the effect of the tastes of the drink, $F(1,92)=0.13, p=.49, \eta_{p}{ }^{2}$ $=.00$. The effect of the types of personality was not significant either, $F(1,92)=0.09, p=.49, \eta_{p}{ }^{2}=.00$, but the interaction between the tastes of the drink and the types of personality was significant, $F(1,92)=13.04, p<0.01, \eta_{p}{ }^{2}$ $=.12$.

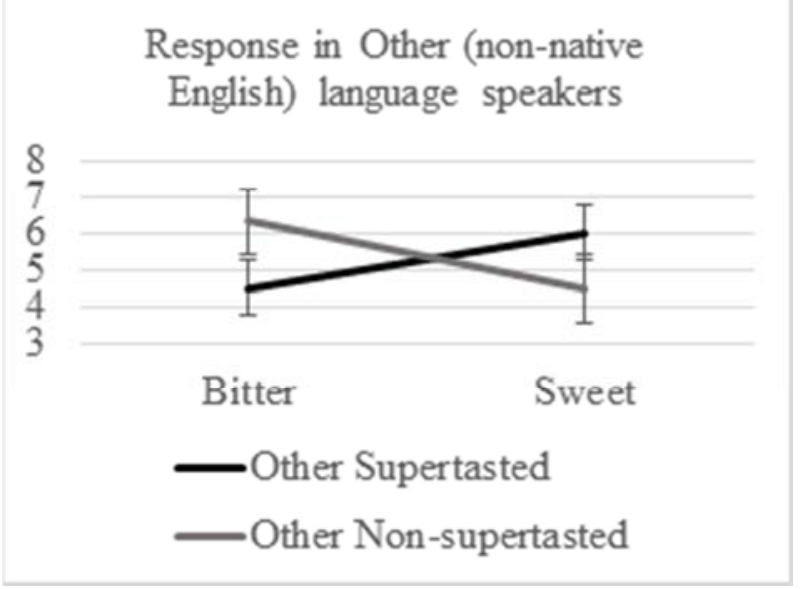

Figure 3. The effect of bitter and sweet drink and cue responses on nonnative English speakers' GRE score performance.

\section{Conclusion}

The findings do not support the hypothesis that selfproduced cue dominant individuals (supertasters) will perform better on cognitive tests after drinking a bitter placebo, and situational cue dominant individuals (nonsupertasters) will perform better on cognitive tests after drinking sweet placebos. The result just reveals the interaction between types of participants and the taste of placebo. Within the native English speakers, the results suggest that the supertasters performed better on cognitive tests after drinking a bitter placebo, and non-supertaster performed better on cognitive tests after drinking a sweet placebo. However, the result of non-native English participants was completely opposite. The result between native English language participants and non-native English language participants are different when they were taking different flavors of placebo, which implies the cultural taste and food preference differences. Western people were exposed to sweet since childhood, which promotes a large consumption of sweet food and increase the concentration of sugar in food and drink in their later lives, such as ice cream, canned fruit and soft drink [22]. Because of that, North Americans do not eat a lot of fruits and vegetables, such as broccoli, Brussels sprouts, and cabbage, which are food that linked to their bitter taste [23]. Generally, people from places where are less affected by the American-like/ Western cultures are less likely to avoid the bitter taste of food. For example, Chinese idiom encourages "bitter taste medicines are better/ more effective" concept. Chinese believes bitter food is healthy and they prefer less sugary, as bitter food can remove internal heat from the body (e.g., bitter melon and bitter greens for treating illness with high fever) [24]. Simultaneously, Indians also have a high preference for bitter and sour tastes [25]. When a value or habit is heavily centralized to a particular preference or practice, the in-group 
members are highly influenced by this particular value or habit. In a word, people will adopt the bitter or sour taste heavily if their communities highly promote bitter- and sourtasting food in the diet. Moskowitz, Kumaraiah, Sharma, Jacobs, and Sharma's study (1975) proved that Indian Karnataka laborers rated citric acid more pleasant when its concentration increased, but Western participants were unpleasant whenever the taste was sour [25]. Thus, cultural influences contribute to the taste and food preferences. As Bloch (1995) said that individuals taste preference can be significantly shaped by the designed community, mass media, and marketers if they are promoting a particular style [26]. When people often consume bitter food in their childhood, daily experience, social norms, and socialization, they will develop a particular taste preference, food preference, and diet habit [27]. Therefore, people will accept more similar tastes and food to their cultures. Since the bitter chamomile tea comes from Asian area and Asians adapt to the bitterness of the tea, the tea may not taste gross to Asian participants, but to Western participants. Conversely, the sweeten chamomile tea may taste gross to Asians but not to Westerners. Cultural taste differences are truly influencing people's perceptions towards bitterness.

Individuals have their own taste preferences on bitterness which caused the different levels of aversion individuals experienced. The taste on the bitterness requires more times to reach maximum intensity in the oral cavity, its duration takes longer than sweetness to return to the baseline [23], and the aftertaste is the long duration of taste after people consuming food or drink. The bitter aftertaste lingers for a longer time than sweet aftertaste in people's oral cavity, which triggers people's aversion of bitterness [28]. Because of the high intensity and long aftertaste with bitterness, people dislike bitter food and drinks. As a result, increasing the bitterness on beverages with repeated ingestion and higher perceived intensity will negatively impact their acceptance, and bitter-tasted beverages are not desirable for consumers [23], [29], [30]. Hence, chamomile tea may be averse to the longer bitter aftertaste.

Besides, biological and genetic factors are also other factors that may affect individual's taste preference. According to Keskitalo, Tuorila, Spector, Cherkas, Knaapila, Silventoinen, and Perola's (2007) study, "liking for sweet foods" and "use-frequency of sweet foods" were mainly contributed to the genetic factor [31], [38]. Genes also matter on bitterness too. The sensitivity to the bitter taste of propylthiouracil (PROP), which investigates individual differences in taste perception, is associated with the liking and acceptance on food and beverages [23], [32], [33], [34], [35]. People who perceived PROP intensely are the supertasters. Supertasters can distinguish small changes in ingredient levels in a forced choice task, they have greater acuity and intensity on tastes than non-supertasters [16]. Because of the specialty of supertasters, they would avoid oily and overly sweet food, and prefer more healthpromoting food and beverages even with a bitter taste [17], [36], [37]. Thus, this may be the reason for them to have the higher tolerance of bitterness and accept bitter chamomile tea for maintaining a healthy status.

To conclude, the concept of "bitter taste medicines are better/ more effective" is not working for every individual, it only works for two types of people: supertasters and people who feel gross after drinking the drink. If individuals are supertasters, they will agree that the aversion from the bitter drink can keep their body healthy. On the other hand, if they are self-motivated people, they will convince themselves the drink does work better for them when they taste the drink as gross. Participants' self-belief on the benefit of bitter drink can change their motivation, behavior, and performances. Overall, the supertasters and taste preferences matter on the placebo the most.

In this study, participants were not being asked about their taste perceptions of their drinks. Although the sweetened tea was sweeter than the unsweetened tea, some individuals may not taste as sweet as others taste like, or they may taste a sweeter taste than others. The cultural adaptation to the bitter and sweetened chamomile tea may also contribute to the differences between native English and non-native English language participants. These differences of personal and cultural taste perception can affect the outcome of the study. For the improvement in the present study, a self-report of taste preference's rating scale should be added in order to accurately group people who have same levels of taste. Future studies should reframe the methods by using a rating scale of placebo taste and a universal flavor on a different placebo substance for participants to perceive the bitterness more easily.

\section{References}

[1] Levine, J., Gordon, N., \& Fields, H. (1978). The mechanism of placebo analgesia. The Lancet, 312 (8091), 654-657.

[2] Shapiro, A. K. (1960). A contribution to a history of the placebo effect. Behavioral Science, 5 (2), 109-135.

[3] Beecher, H. K. (1955). The powerful placebo. Journal of the American Medical Association, 159 (17), 1602-1606.

[4] Cabot, R. C. (1906). The physician's responsibility for the nostrum evil. Journal of the American Medical Association, 47 (13), 982-984.

[5] Kienle, G. S., \& Kiene, H. (1997). The powerful placebo effect: fact or fiction? Journal of clinical epidemiology, 50 (12), 1311-1318.

[6] Ernst, E., \& Resch, K. L. (1995). Concept of true and perceived placebo effects. BMJ: British Medical Journal, 311 (7004), 551.

[7] Waber, R. L., Shiv, B., \& Carmon, Z. (2008). Commercial features of placebo and therapeutic. Jama, 299 (9), 1016-7.

[8] Lasagna, L., Laties, V. G., \& Dohan, J. L. (1958). Further studies on the "pharmacology" of placebo administration. Journal of Clinical Investigation, 37 (4), 533.

[9] Schindel, L. (1978). The placebo dilemma. European journal of clinical pharmacology, 13 (3), 231-235. 
[10] Jacobs, K. W., \& Nordan, F. M. (1979). Classification of placebo drugs: effect of color. Perceptual and motor skills, 49 (2), 367-372.

[11] Marchand, S., \& Arsenault, P. (2002). Odors modulate pain perception: a gender-specific effect. Physiology \& Behavior, $76(2), 251-256$.

[12] Eccles, R. (2006). Mechanisms of the placebo effect of sweet cough syrups. Respiratory Physiology \& Neurobiology, 152 (3), 340-348.

[13] Huffman, M. A. (1997). Current evidence for self-medication in primates: a multidisciplinary perspective. American Journal of Physical Anthropology, 104 (s 25), 171-200.

[14] Mennella, J. A., Spector, A. C., Reed, D. R., \& Coldwell, S. E. (2013). The bad taste of medicines: overview of basic research on bitter taste. Clinical therapeutics, 35 (8), 12251246.

[15] Mennella, J. A., Pepino, M. Y., \& Reed, D. R. (2005). Genetic and environmental determinants of bitter perception and sweet preferences. Pediatrics, 115 (2), e216-e222.

[16] Hayes, J. E., \& Keast, R. S. (2011). Two decades of supertasting: where do we stand? Physiology \& Behavior, 104 (5), 1072-1074.

[17] Wilkie, L. M. (2012). Individual Differences in Taste Perception and Bitterness Masking (Doctoral dissertation, Arizona State University).

[18] Duncan, J. W. \& Laird, J. D. (1980). Positive and negative placebo effects as a function of differences in cues used selfperception. J. Pers. Sot. Psychof., 39, 1024-36.

[19] Stevens, D. A. (1996). Individual differences in taste perception. Food Chemistry, 56 (3), 303-311.

[20] James, W. (1884). II.-What is an emotion? Mind, 34, 188205.

[21] Bern, D. J. (1987). Self-perception: An alternative interpretation of cognitive dissonance. Psychol. Rev., 74, 183200.

[22] Drewnowski, A. (1997). Taste preferences and food intake. Annual Review of Nutrition, 17 (1), 237-253.

[23] Gaudette, N. J., \& Pickering, G. J. (2013). Modifying bitterness in functional food systems. Critical Reviews in Food Science and Nutrition, 53 (5), 464-481.

[24] Weng, W., \& Chen, J. (1996). The eastern perspective on functional foods based on traditional Chinese medicine. Nutrition Reviews, 54 (11), S11.

[25] Moskowitz, H. W., Kumaraiah, V., Sharma, K. N., Jacobs, H. L., \& Sharma, S. D. (1975). Cross-cultural differences in simple taste preferences. Science, 190 (4220), 1217-1218.
[26] Bloch, P. H. (1995). Seeking the ideal form: product design and consumer response. Journal of Marketing, 59 (3), 16-29.

[27] Tiu Wright, L., Nancarrow, C., \& Kwok, P. M. (2001). Food taste preferences and cultural influences on consumption. British Food Journal, 103 (5), 348-357.

[28] Sami, P. S., Toma, R. B., Nelson, D. B., \& Frank, G. C. (1997). Effects of debittering on grapefruit juice acceptance. International Journal of Food Sciences and Nutrition, 48 (4), 237-242.

[29] Drewnowski, A., \& Gomez-Carneros, C. (2000). Bitter taste, phytonutrients, and the consumer: a review. The American Journal of Clinical Nutrition, 72 (6), 1424-1435.

[30] Lesschaeve, I., \& Noble, A. C. (2005). Polyphenols: factors influencing their sensory properties and their effects on food and beverage preferences. The American journal of Clinical Nutrition, 81 (1), 330S-335S.

[31] Keskitalo, K., Tuorila, H., Spector, T. D., Cherkas, L. F., Knaapila, A., Silventoinen, K., \& Perola, M. (2007). Same genetic components underlie different measures of sweet taste preference. The American journal of clinical nutrition, 86 (6), 1663-1669.

[32] Bartoshuk, L. M., Duffy, V. B., \& Miller, I. J. (1994). PTC/PROP tasting: anatomy, psychophysics, and sex effects. Physiology \& Behavior, 56 (6), 1165-1171.

[33] Drewnowski, A., Henderson, S. A., \& Shore, A. B. (1997). Taste responses to naringin, a flavonoid, and the acceptance of grapefruit juice are related to genetic sensitivity to 6-npropylthiouracil. The American Journal of Clinical Nutrition, 66 (2), 391-397.

[34] Tepper, B. J. (1998). 6-n-Propylthiouracil: a genetic marker for taste, with implications for food preference and dietary habits. The American Journal of Human Genetics, 63 (5), 1271-1276.

[35] Duffy, V. B., \& Bartoshuk, L. M. (2000). Food acceptance and genetic variation in taste. Journal of the American Dietetic Association, 100 (6), 647-655.

[36] Bartoshuk, L. M. (1979). Bitter taste of saccharin related to the genetic ability to taste the bitter substance 6-npropylthiouracil. Science, 205 (4409), 934-935.

[37] Tepper, B. J., \& Nurse, R. J. (1998). PTC taster status is related to fat perception and preference. Annals of the New York Academy of Sciences, 885 (1), 802- 804.

[38] Perola, M. (2007). Same genetic omponents underlie different measures of sweet taste preference. The American Journal of Clinical Nutrition, 86 (6), 1663-1669.

[39] Dowdey, S. (2007). How taste works. How Stuff Works. 